\title{
MINI-REVIEW
}

\section{Clinical Efficacy and Possible Applications of Genomics in Lung Cancer}

\author{
Khalid Khalaf Alharbi*
}

\begin{abstract}
The heterogeneous nature of lung cancer has become increasingly apparent since introduction of molecular classification. In general, advanced lung cancer is an aggressive malignancy with a poor prognosis. Activating alterations in several potential driver oncogenic genes have been identified, including EGFR, ROS1 and ALK and understanding of their molecular mechanisms underlying development, progression, and survival of lung cancer has led to the design of personalized treatments that have produced superior clinical outcomes in tumours harbouring these mutations. In light of the tsunami of new biomarkers and targeted agents, next generation sequencing testing strategies will be more appropriate in identifying the patients for each therapy and enabling personalized patients care. The challenge now is how best to interpret the results of these genomic tests, in the context of other clinical data, to optimize treatment choices. In genomic era of cancer treatment, the traditional one-size-fits-all paradigm is being replaced with more effective, personalized oncologic care. This review provides an overview of lung cancer genomics and personalized treatment.
\end{abstract}

Keywords: Lung cancer - genomics - personalized treatment

Asian Pac J Cancer Prev, 16 (5), 1693-1698

\section{Introduction}

Regardless of histologic subtype, lung cancer is one of the most genomically diverse and unstable of all cancers, creating terrific challenges for both prevention and treatment strategies (Larsen and Minna, 2011; DeSantis et al., 2012). Nevertheless, this same biologic diversity provides a number of opportunities for utilization of interpatient tumor heterogeneity by ungrouping lung cancer into a variety of molecularly defined subsets for which mutations and/or abnormal gene expressions drive disease progression, survival and can serve as druggable targets (Sun et al., 2007; Ding et al., 2008; Pao and Girard, 2011).

Although the transition from traditional to mechanismbased, molecular marker driven therapeutic decision making is in its early phases, new drug classes have already changed the paradigm for the management of advanced-stage lung cancer (Gandara et al., 2009; 2012). A well studied example is the gain-of-function tyrosine kinase activating epidermal growth factor receptor (EGFR) mutations as the best predictive biomarker over clinicopathologic features in predicting tumor response and progression free survival to EGFR tyrosine kinase inhibitors (TKIs) (Mok et al., 2009; Mitsudomi etal., 2010; Maemondo., 2010; Zhou et al., 2011; Rosell et al ., 2012). Similarly, gain-of-function tyrosine kinase activating ALK gene rearrangements, strong predictive biomarker in predicting tumor response and progression free survival to the first-in-class ALK TKI crizotinib (Kwak et al., 2010). US FDA granted approval of the first-in-class ALK inhibitor crizotinib for treatment of ALK-positive advanced NSCLCs (US Food and Drug Administration). Subsequently, both the National Comprehensive Cancer Network (NCCN) and American Society of Clinical Oncology guidelines recommended EGFR mutation and ALK gene rearrangements testing on all NSCLCs that contain an adenocarcinoma component, regardless of histologic grade or dominant histologic subtype (Keedy et al ., 2011; National Comprehensive Cancer Network, 2015). EGFR mutation and ALK testing is not recommended for pure squamous cell carcinomas, pure small-cell carcinomas, or pure neuroendocrine carcinomas (Field et al., 2011).

Although clinical utility of single gene biomarker has already proven successful in guiding selection of targeted therapies in non small cell lung cancer (NSCLC) (Von et al., 2010; Paik et al., 2012). Recent genomic studies have revealed that distinct genomic abnormalities are present in adenocarcinomas and squamous cell carcinomas (Jemal et al., 2011; Govindan et al., 2012), providing opportunities for developing novel molecularly targeted and biomarker driven therapeutic strategies for specific molecular subsets of patients.

Several high throughput studies identified new susceptibility loci for lung cancer, which highlights the genetic heterogeneity of lung cancer susceptibility among different ethnic populations (Brian et al., 2012). However, 
most of studies of genome-wide alterations in lung cancer have been carried out in patients from Western countries, and to our best knowledge none of such studies have been done in Asian population. Moreover, individual tumors vary widely in evolution and behavior (i.e., tumor dormancy, local growth, distant dissemination, treatment response, and relapse). Thus, individuals who share the same histopathological stages, grades, and receive the same treatments could have tumors with completely different evolutionary histories and clinical outcomes. Moreover, some patients with non-aggressive disease can live over 5 years, whereas others die from metastatic disease within 2-3 years after diagnosis. Several lines of evidence have suggested that the heterogeneous clinical behavior of lung cancer is the result of different underlying molecular mechanisms during tumor progression (Jemal et al., 2010).

\section{Shift from Microarrays to Next Generation Sequencing}

Microarray technology has been invaluable tools for studying of complex biological mechanisms, resulting in a new understanding of lung cancer pathogenesis and providing a foundation for the generation of novel biomarkers for diagnosis, prognosis and the prediction of treatment responses. Although microarray based studies have contributed significantly to our understanding of the development and progression of human cancer, these technologies have several major limitations, including their inability to detect structural genomic aberration, alternative splice mechanism etc. The rapid development of next-generation sequencing (NGS) technologies has overcome many of these problems (Morozova and Marra 2008; Voelkerding et al., 2009; Wang et al., 2009). NGS permits the investigation of an entire cancer genome and transcriptome with unprecedented resolution and throughput (Bell, 2010; Ding et al., 2010; Ozsolak and Milos, 2011). The first whole cancer genome sequence was reported in 2008, which compared the DNA sequence from an acute myeloid leukemia with that from normal skin from the same patient (Meyerson et al., 2010). Subsequently, whole-genome sequencing has been used to identify a wide range of genomic alterations, such as nucleotide substitutions, copy number alterations and structural rearrangements in lung, breast, prostate and pancreatic cancers, ovarian carcinoma, leukemia and melanoma (Ley et al., 2008; Mardis et al., 2009; Campbell et al., 2010; Ding et al 2010; Jones et al., 2010; Lee et al., 2010; Ley et al., 2010; Pleasance et al., 2010). Sequencing of whole transcriptome (RNA-seq) is highly sensitive and efficient method for detecting gene fusions, somatic mutations and alternatively spliced forms (Stephens et al., 2009). For example, a comparison of fluorouracilresistant and -nonresistant human colorectal cancer cell lines revealed a global disruption of splicing in the fluorouracil-resistant cells, which was characterized by the expression of new mRNA isoforms resulting from exon skipping, alternative splice site usage and intron retention (Griffith et al., 2010). Novel somatic mutations have been discovered by RNA-seq in granulose cell tumors of the ovary and endometriosis-associated ovarian carcinomas (Shah et al., 2009; Wiegand et al., 2010). Paired-end RNA-seq has been mainly used to comprehensively elucidate gene fusion products in cancer transcriptomes (Maher et al., 2009; Zhao et al., 2009). These preliminary studies have led to the comprehensive discovery of novel alterations in the cancer genome and new insights into the pathogenesis of cancer.

\section{Prognostic and Predictive Molecular Signatures}

For centuries its well known that medication to a given disease yields different and varied response in different patients. Even there are cases where there is no response at all. Hence, the question is: Why is this variation in response seen to the same standardized and medically approved treatment strategies? One of the recent studies has demonstrated that miRNA targeting can be used to review the clinical efficacy and resistant mechanism of various cancers (Zhou et al ., 2014). Now in the genomic era, we know that the probable cause is the difference in the genomic makeup of different individuals which may be responsible for a wide range of treatment response. With the completion of the Human Genome project and the development of molecular biology techniques, genomic tests now exist to answer some of these questions. Subsequently, number of potential therapeutic targets involved in disease driving molecular pathways was unveiled (Figure 1), drastically altering the clinical evaluation and treatment of patients. By examining the genomic differences between individuals, we can understand the impact of diagnosis, prognosis and treatment. The expanding knowledge of the molecular basis of cancer has shown that variation in gene expression patterns can guide therapy not only for treating neoplastic condition but can prognostic and predictive markers.

Several published clinical practice guidelines by the National Comprehensive Cancer Network (NCCN), US

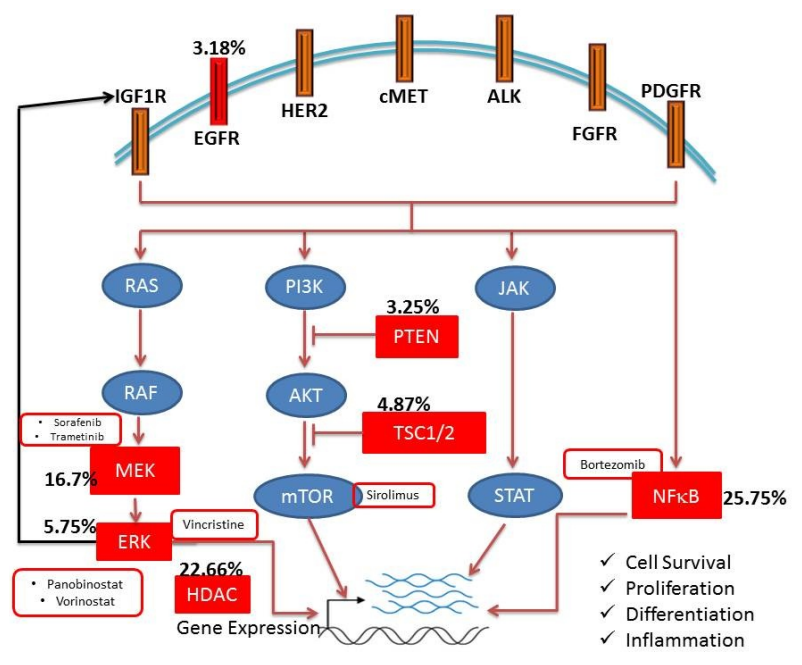

Figure 1. Showing Number of Potential Therapeutic Targets Involved in Disease Driving Molecular Pathways 
FDA and the American Society of Clinical Oncology (ASCO), now recommend that all patients with NSCLC with adenocarcinoma component undergo testing for EGFR mutations and ALK rearrangements (Keedy et al., 2011; Lindeman et al., 2013) NCCN (Version 1.2015). The identification and characterization of molecular targets are having a growing impact on the management of patients with lung cancer.

Prognostic and predictive molecular signatures in surgically resected lung cancer have been developed by several research groups (Zhu et al., 2010; Kratz et al., 2013; Tang et al 2013). However, the reproducibility, cost, and lack of validation of these molecular signatures often hinder the clinical application. Zhu et al. (2010) developed a 15-gene expression panel that showed stage IB/II NSCLC patients are most likely to benefit from adjuvant chemotherapy with cisplatin/vinorelbine. Similarly, Kratz et al. (2013) developed a prognostic gene signature that was able to identify patients with early-stage NSCLC at high risk for mortality after surgical resection. More recently, Tang et al. (2013) developed an 18-gene prognostic panel in resectable NSCLC, which was then incorporated with genome-wide functional data and genetic aberration data to develop a 12-gene predictive panel for survival benefits with adjuvant chemotherapy .

\section{Driver Oncogenic Genes and their role in Personalized Treatment}

Few examples of driver oncogenes genes in lung cancer were considered if it is genetically activated in lung cancer and if there is clinically approved inhibitor or convincing proof-of-concept data that suggests high response rates in a targeted population.

\section{EGFR}

Mutations of the EGFR gene are a well-established example of an oncogenic driver in NSCLC subtype of lung cancer. EGFR activating mutations are found in $~ 10 \%$ and $\sim 40 \%$ of NSCLCs in Caucasians and Asian patients respectively, and are primarily seen in adenocarcinomas (Sos et al., 2009). Several platforms are used to study EGFR mutations from tumour tissue specimens. Gefitinib, EGFR inhibitor is also approved as monotherapy for EGFR mutation-positive NSCLC following failure of platinumand docetaxel-based chemotherapy. Another EGFR TKI afatinib recently gained FDA approval as first-line therapy for EGFR mutation-positive NSCLC. Similarly, dacomitinib-EGFR TKI, demonstrated preclinical efficacy in NSCLC tumours harbouring the T790M gatekeeper mutation (Engelman et al., 2007; Gonzales et al., 2008), found in $\sim 50 \%$ of NSCLCs that have acquired resistance to erlotinib or gefitinib (Kobayashi et al., 2005; Pao et al., 2005).

\section{ALK}

ALK is a transmembrane tyrosine-kinase receptor expressed in the small intestine, testes, and brain, but not normally in the lung. Recently ALK gene rearrangements have been identified as oncogenic drivers in NSCLC subtype of lung cancer. ALK signaling is activated by the creation of oncogenic fusions of the ALK gene with EML4 (Soda et al., 2007), although other fusion partners exist . ALK-EML4 rearrangements are found in $~ 7 \%$ of NSCLC patients (Koivunen et al., 2008; Kwak et al., 2010), usually in young never-smokers with adenocarcinoma (Rodig et al., 2009; Shaw et al., 2009; 2011; Sasaki et al., 2010). Tumours with ALK gene rearrangements are resistant to the EGFR TKIs gefitinib and erlotinib (Shaw et al., 2009).

\section{ROS1}

ROS1 is a tyrosine-kinase receptor of the insulin receptor family. ROS1 gene rearrangements are well known oncogenic drivers in NSCLC subtype of lung cancer, and several fusion patterns have been identified (Bergethon et al., 2012; Rimkunas et al., 2012). ROS1 fusions are often seen in young never-smokers with adenocarcinoma, a population similar to those with ALK-rearranged NSCLC (Bergethon et al., 2012). ROS1 rearrangements rarely present simultaneously with EGFR, ALK or KRAS alterations (Gainor et al., 2013).

Crizotinib has shown nearly complete response in a patient with advanced ROS1-positive NSCLC in clinical trial (Bergethon et al., 2012). In an expansion cohort of the trial, 14 patients received crizotinib for ROS1-rearranged NSCLC and nine (64\%) had a confirmed response (Shaw et al., 2012). A further case of a complete metabolic response to crizotinib was reported in a patient with advanced ROS1-positive NSCLC . A ROS1 monoclonal antibody (D4D6) has recently been developed and validated for use in IHC assays (Rimkunas et al., 2012).

\section{PTEN}

Deleterious mutation of PTEN and loss of PTEN protein expression are common in lung cancer (Marsit et al., 2005) which plays a significant role in cell cycle progression, apoptosis, proliferation, and migration via negative regulation of the PI3K/Akt/mTOR pathway (Abdulkareem et al., 2013). PTEN loss of function mutation has also been linked with acquired resistance to EGFR TKIs in EGFR mutation-positive NSCLC (Sos et al., 2009). The TKI vandetanib has shown efficacy against EGFR mutation-positive lung cancer showing PTEN deleterious mutation, suggesting that it may also be effective in patients with EGFR mutation-positive NSCLC whose tumours lack PTEN expression (Takeda et al., 2013). However, in many cases, the functional consequences of PTEN mutations remain to be elucidated.

\section{PI3K}

PI3Ks are lipid kinases involved in the regulation of cell proliferation, growth, and survival. Deleterious mutations in the PIK3CA gene that encodes subunit of PI3K $\alpha$ have been identified in several cancers (Samuels et al., 2004). Additionally, aberrant signaling via PI3K/ Akt/mTOR pathway has been observed in a number of human cancers, including lung cancer (Trigka et al .,2013). 
PIK3CA mutations often co-exist with other oncogenic mutations, particularly EGFR, ALK, KRAS (Chaft et al., 2012). In addition, PI3K dependency in some tumours, is seen due to PTEN loss of function (Pfeifer et al., 2013). Several PI3K inhibitors are in clinical trials, but the response rate to single agents has to be yet (Pao et al., 2011; Thomas et al., 2013).

\section{Conclusions}

A deeper understanding of the molecular classification of lung cancer may ultimately lead to personalized treatment strategies, which will improve care for those patients most likely to benefit, and spare the cost and morbidity associated with failed treatment interventions. Multiplex PCR assays, high-throughput technologies such as NGS, and hopefully some form of multiplex protein-based platform will play an important role in lung carcinoma management and rational therapy selection, but there are many challenges ahead. Careful design of clinical trials will help to evaluate molecularly targeted agents in the context of those populations most likely to benefit, but clinicians will be faced with difficult decisions, such as how to include an ethically fair control arm, what treatment to choose when a new patient subset is no longer part of the first-line population, and what the preferential order of treatment should be where multiple molecular targets are present. Only through a better understanding of the disease can treatment choices be enhanced and the outlook for patients with lung cancer improved.

Although previous studies have comprehensively evaluated lung cancer at molecular level by RNA-seq, to our best knowledge none of these studies were performed with Asian population. While RNA-seq permits the simultaneous analysis of gene expression, noncoding RNA (ncRNA) expression, alternative splicing, somatic mutations and gene fusions, no systematic analyses of lung cancer transcriptomic data have been reported. RNA-seq simultaneously reveals multiple aspects of the transcriptome, including gene fusions, alternative splicing, the expression of long ncRNAs and genes, and somatic mutations.

Furthermore, characterize the global transcriptional changes in lung cancer samples by comparing them with normal, and therefore, it will be a new revolutionary direction in the pathogenesis of lung cancer biology as it would help more efficient diagnosis and drastically enhance the specificity of treatment. This study will unravel a complex landscape of functional genomic alterations in lung cancer, which will delineate concept of lung cancer heterogeneity and advance our understanding of this disease in the Indian population. Development of molecular signatures for lung cancer diagnosis if successful can prove to be path-breaking in uplifting the diagnosis and therapeutic sector of lung cancer.

\section{Acknowledgements}

The author is thankful to the Department of Laboratory Sciences, College of Applied Medical sciences, King Saud University, for encouragement and providing facilities for carrying out present review. Thanks are also due to Dr.Rabbani Syed and Dr. Imran Ali Khan for their valuable support in compiling the review .

\section{References}

Abdulkareem IH, Blair M (2013). Phosphatase and tensin homologue deleted on chromosome 10. Niger Med J, 54, 79-86.

Brian E, Henderson, Norman H, Lee, Victoria Seewaldt, Hongbing Shen (2012). The influence of race and ethnicity on the biology of cancer. Nature Reviews Cancer, 12, 648-53.

Bergethon K, Shaw AT, Ou SH, et al (2012). ROS1 rearrangements define a unique molecular class of lung cancers. J Clin Oncol, 30, 863-70.

Bell DW (2010). Our changing view of the genomic landscape of cancer. J Pathol, 220, 231-43.

Campbell PJ, Yachida S, Mudie LJ, et al (2010). The patterns and dynamics of genomic instability in metastatic pancreatic cancer. Nature, 467, 1109-13.

Chaft JE, Arcila ME, Paik PK, et al (2012). Coexistence of PIK3CA and other oncogene mutations in lung adenocarcinoma: rationale for comprehensive mutation profiling. Mol Cancer Ther, 11, 485-91.

Ding L, Wendl MC, Koboldt DC, Mardis ER (2010). Analysis of next-generation genomic data in cancer: accomplishments and challenges. Hum Mol Genet, 19, 188-96.

Ding L, Ellis MJ, Li S, et al (2010). Genome remodelling in a basal-like breast cancer metastasis and xenograft. Nature, 464, 999-1005.

DeSantis C, Virgo K, et al (2012). Cancer treatment and survivorship statistics, 2012. CA Cancer J Clin, 62, 220-41

Ding L, Getz G, Wheeler DA, et al (2008). Somatic mutations affect key pathways in lung adenocarcinoma. Nature, $\mathbf{4 5 5}$, 1069-75.

Engelman JA, Zejnullahu K, Gale CM, et al (2007). PF00299804, an irreversible pan-ERBB inhibitor, is effective in lung cancer models with EGFR and ERBB2 mutations that are resistant to gefitinib. Cancer Res, 67, 11924-32.

Field JK, Brambilla C, Caporaso N, et al (2002). Consensus statements from the second international lung cancer molecular biomarkers workshop: a European strategy for developing lung cancer molecular diagnostics in high risk populations. Int J Oncol, 21, 369-73.

Gandara DR, Mack PC, Li T, et al (2009). Evolving treatment algorithms for advanced non-small-cell lung cancer: 2009 looking toward 2012. Clin Lung Cancer, 10,392-4.

Gandara DR, Li T, Lara PN, Jr, et al (2012). Algorithm for codevelopment of new drug-predictive biomarker combinations: Accounting for inter- and intrapatient tumor heterogeneity. Clin Lung Cancer, 13, 321-5.

Gonzales AJ, Hook KE, Althaus IW, et al (2008). Antitumor activity and pharmacokinetic properties of PF-00299804, a second-generation irreversible pan-erbB receptor tyrosine kinase inhibitor. Mol Cancer Ther, 7, 1880-9.

Gainor JF, Shaw AT (2013). Novel targets in non-small cell lung cancer: ROS1 and RET fusions. Oncologist, 18, 865-75.

Govindan R, Hammerman PS, Hayes DN, et al (2012). Comprehensive genomic characterization of squamous cell carcinoma of the lung. J Clin Oncol, 30,453s. abstr 7006.

Griffith M, Griffith OL, Mwenifumbo J, et al (2010). Alternative expression analysis by RNA sequencing. Nat Methods, 7 , 843-7.

Jemal A, Bray F, Center MM, Ferlay J, Ward E, Forman D (2011). Global cancer statistics. CA Cancer J Clin, 61, 69-90.

Jemal A, Siegel R, Xu J, Ward E (2010). Cancer statistics. $C A$ Cancer J Clin, 60, 277-300. 
Jones S, Wang TL, Shih Ie M, et al (2010). Frequent mutations of chromatin remodeling gene ARID1 A in ovarian clear cell carcinoma. Science, 330, 228-31.

Kratz JR, He J, Van Den Eeden SK, et al (2012). A practical molecular assay to predict survival in resected nonsquamous, non-small-cell lung cancer: development and international validation studies. Lancet, 379, 823-32.

Kobayashi S, Boggon TJ, Dayaram T, et al (2005). EGFR mutation and resistance of non-small-cell lung cancer to gefitinib. N Engl J Med, 352, 786-92.

Koivunen JP, Mermel C, Zejnullahu K, et al (2008). EML4-ALK fusion gene and efficacy of an ALK kinase inhibitor in lung cancer. Clin Cancer Res, 14, 4275-83.

Kwak EL, Bang YJ, Camidge DR, et al (2010). Anaplastic lymphoma kinase inhibition in non-small-cell lung cancer. N Engl J Med, 363, 1693-703.

Keedy VL, Temin S, Somerfield MR, et al (2011). American society of clinical oncology provisional clinical opinion: epidermal growth factor receptor (EGFR) mutation testing for patients with advanced non-small-cell lung cancer considering first-line EGFR tyrosine kinase inhibitor therapy. J Clin Oncol, 29, 2121-7.

Keedy VL, Temin S, Somerfield MR, et al (2011). American society of clinical oncology provisional clinical opinion: Epidermal growth factor receptor (EGFR) mutation testing for patients with advanced non-small-cell lung cancer considering first-line EGFR tyrosine kinase inhibitor therapy. J Clin Oncol, 29, 2121-7.

Kwak EL, Bang YJ, Camidge DR, et al (2010). Anaplastic lymphoma kinase inhibition in non-small-cell lung cancer. N Engl J Med, 363, 1693-703.

Ley TJ, Mardis ER, Ding L, et al (2008). DNA sequencing of a cytogenetically normal acute myeloid leukaemia genome. Nature, 456, 66-72.

Lindeman NI, Cagle PT, Beasley MB, et al (2013). Molecular testing guideline for selection of lung cancer patients for EGFR and ALK tyrosine kinase inhibitors: guideline from the college of American pathologists, international association for the study of lung cancer, and association for molecular pathology. J Thorac Oncol, 8, 823-59.

Lee W, Jiang Z, Liu J, et al (2010). The mutation spectrum revealed by paired genome sequences from a lung cancer patient. Nature, 465, 473-7.

Ley TJ, Ding L, Walter MJ, et al (2010). DNMT3A mutations in acute myeloid leukemia. $N$ Engl J Med, 363, 2424-33.

Larsen JE, Minna JD (2011). Molecular biology of lung cancer: clinical implications. Clin Chest Med, 32, 703-40.

Morozova O, Marra MA(2008). Applications of next-generation sequencing technologies in functional genomics. Genomics, 92, 255-64.

Maher CA, Palanisamy N, Brenner JC, et al (2009). Chimeric transcript discovery by paired-end transcriptome sequencing. Proc Natl Acad Sci USA, 106, 12353-8.

Meyerson M, Gabriel S, Getz G (2010). Advances in understanding cancer genomes through second-generation sequencing. Nat Rev Genet, 11, 685-96.

Mardis ER, Ding L, Dooling DJ, et al (2009). Recurring mutations found by sequencing an acute myeloid leukemia genome. N Engl J Med, 361, 1058-66.

Mok TS, Wu YL, Thongprasert S, et al (2009). Gefitinib or carboplatin-paclitaxel in pulmonary adenocarcinoma. $N$ Engl J Med, 361, 947-57.

Mitsudomi T, Morita S, Yatabe Y, et al (2010). Gefitinib versus cisplatin plus docetaxel in patients with non-small-cell lung cancer harbouring mutations of the epidermal growth factor receptor (WJTOG3405): An open label, randomised phase 3 trial. Lancet Oncol, 11, 121-8.
Maemondo M, Inoue A, Kobayashi K, et al (2010). Gefitinib or chemotherapy for non-small-cell lung cancer with mutated EGFR. N Engl J Med, 362, 2380-8.

Marsit CJ, Zheng S, Aldape K, et al (2005). PTEN expression in non-small cell lung cancer: evaluating its relation to tumor characteristics, allelic loss, and epigenetic alteration. Hum Pathol, 36, 768-76.

National Comprehensive Cancer Network: Non-Small Cell Lung Cancer. Version 1.2015. NCCN clinical practice guidelines in oncology. http://www.ncen.com.

Ozsolak F, Milos PM (2011). RNA sequencing: advances, challenges and opportunities. Nat Rev Genet, 12, 87-98.

Paik PK, Hasanovic A, Wang L, et al (2012). Multiplex testing for driver mutations in squamous cell carcinomas of the lung. J Clin Oncol, 30, 481s. abstr 7505.

Pleasance ED, Cheetham RK, Stephens PJ, et al (2010). A comprehensive catalogue of somatic mutations from a human cancer genome. Nature, 463, 191-6.

Pao W, Miller VA, Politi KA, et al (2005). Acquired resistance of lung adenocarcinomas to gefitinib or erlotinib is associated with a second mutation in the EGFR kinase domain. PLoS Med, 2, 73 .

Pao W, Girard N (2011). New driver mutations in non-small-cell lung cancer. Lancet Oncol, 12, 175-80.

Pfeifer M, Grau M, Lenze D, et al (2013). PTEN loss defines a PI3K/AKT pathway-dependent germinal center subtype of diffuse large B-cell lymphoma. Proc Natl Acad Sci USA, 110, 12420-5.

Rosell R, Carcereny E, Gervais R, et al (2012). Erlotinib versus standard chemotherapy as first-line treatment for European patients with advanced EGFR mutation-positive non-smallcell lung cancer (EURTAC): a multicentre, open-label, randomised phase 3 trial. Lancet Oncol, 13, 239-46.

Rodig SJ, Mino-Kenudson M, Dacic S, et al (2009). Unique clinicopathologic features characterize ALK-rearranged lung adenocarcinoma in the western population. Clin Cancer Res, 15, 5216-23.

Rimkunas VM, Crosby KE, Li D, et al (2012). Analysis of receptor tyrosine kinase ROS1-positive tumors in non-small cell lung cancer: identification of a FIG-ROS1 fusion. Clin Cancer Res, 18, 4449-57.

Sun S, Schiller JH, Spinola M, et al (2007). New molecularly targeted therapies for lung cancer. J Clin Invest, 117, 274050.

Soda M, Choi YL, Enomoto M, et al (2007). Identification of the transforming EML4-ALK fusion gene in non-small-cell lung cancer. Nature, 448, 561-6.

Shaw AT, Yeap BY, Mino-Kenudson M, et al (2009). Clinical features and outcome of patients with non-small-cell lung cancer who harborEML4-ALK. J Clin Oncol, 27, 4247-53.

Shaw AT, Yeap BY, Solomon BJ, et al (2011). Effect of crizotinib on overall survival in patients with advanced non-smallcell lung cancer harbouring ALK gene rearrangement: a retrospective analysis. Lancet Oncol, 12, 1004-12.

Stephens PJ, McBride DJ, Lin ML, et al (2009). Complex landscapes of somatic rearrangement in human breast cancer genomes. Nature, 462, 1005-10.

Shah SP, Kobel M, Senz J, et al (2009). Mutation of FOXL2 in granulosa-cell tumors of the ovary. $N$ Engl J Med, 360, 2719-29.

Sos ML, Koker M, Weir BA, et al (2009). PTEN loss contributes to erlotinib resistance in EGFR-mutant lung cancer by activation of Akt and EGFR. Cancer Res, 69, 3256-61.

Shaw AT, Camidge DR, Engelman JA (2012). Clinical activity of crizotinib in advanced non-small cell lung cancer (NSCLC) harboringROS1 gene rearrangement. J Clin Oncol, 30, 7508.

Sasaki T, Rodig SJ, Chirieac LR, et al (2010). The biology and 
Khalid Khalaf Alharbi et al

treatment of EML4-ALK non-small cell lung cancer. Eur J Cancer, 46, 1773-80.

Samuels Y, Wang Z, Bardelli A, et al (2004). High frequency of mutations of the PIK3CA gene in human cancers. Science, 304, 554 .

Trigka EA, Levidou G, Saetta AA, et al (2013). A detailed immunohistochemical analysis of the PI3K/AKT/mTOR pathway in lung cancer: correlation with PIK3CA, AKT1, K-RAS or PTEN mutational status and clinicopathological features. Oncol Rep, 30, 623-36.

Thomas A, Rajan A, Lopez-Chavez A, et al (2013). From targets to targeted therapies and molecular profiling in non-small cell lung carcinoma. Ann Oncol, 24, 577-85.

Tang H, Xiao G, Behrens C, et al (2013). A 12-gene set predicts survival benefits from adjuvant chemotherapy in non-small cell lung cancer patients. Clin Cancer Res, 19, 1577-86.

Takeda H, Takigawa N, Ohashi K, et al (2013). Vandetanib is effective in EGFR-mutant lung cancer cells with PTEN deficiency. Exp Cell Res, 319, 417-23.

US Food and Drug Administration: FDA approves Xalkori with companion diagnostic for a type of late-state lung cancer. http://www.accessdata.fda.gov/drugsatfda_docs/ appletter/2011/202570s000ltr.pdf

Von Hoff DD, Stephenson JJ, Jr, Rosen P, et al (2010). Pilot study using molecular profiling of patients' tumors to find potential targets and select treatments for their refractory cancers. J Clin Oncol, 20, 4877-883.

Voelkerding KV, Dames SA, Durtschi JD (2009). Nextgeneration sequencing: from basic research to diagnostics. Clin Chem, 55, 641-58.

Wang Z, Gerstein M, Snyder M (2009). RNA-Seq: a revolutionary tool for transcriptomics. Nat Rev Genet, 10, 57-63.

Wiegand KC, Shah SP, Al-Agha OM, et al (2010). ARID1A mutations in endometriosis-associated ovarian carcinomas. N Engl J Med, 363, 1532-43.

Zhou C, Wu YL, Chen G, et al (2011). Erlotinib versus chemotherapy as first-line treatment for patients with advanced EGFR mutation-positive non-small-cell lung cancer (OPTIMAL, CTONG-0802): A multicentre, openlabel, randomised, phase 3 study. Lancet Oncol, 12, 735-42.

Zhao Q, Caballero OL, Levy S, et al (2009). Transcriptomeguided characterization of genomic rearrangements in a breast cancer cell line. Proc Natl Acad Sci USA, 106, 1886-91.

Zhu CQ, Ding K, Strumpf D, et al (2010). Prognostic and predictive gene signature for adjuvant chemotherapy in resected non-small-cell lung cancer. J Clin Oncol, 28, 4417-24.

Zhou YM, Liu J, Sun W (2014). MiR-130a overcomes gefitinib resistance by targeting met in non-small cell lung cancer cell lines. Asian Pac J Cancer Prev, 15, 1391-6. 